\title{
Construction of Probabilistic Atlas and Its Application to Detection of Lacunar Infarcts
}

\author{
H. Tanaka, T. Tateyama, X.H. Han, Y.W. Chen \\ Graduate School of Information Science and Engineering, \\ Ritsumeikan University \\ Shiga, Japan
}

\author{
A. Shiino \\ Biomedical MR Science Center, Shiga University of \\ Medical Science \\ Shiga, Japan
}

\author{
X. Wei \\ Institute for Infocomm Research, A-STAR \\ Singapore 138632
}

\begin{abstract}
In this paper, we propose using probabilistic atlas of lacunar infarcts to improve accuracy of lacunar infarcts detection. The cerebral vascular disease is responsible for about $10 \%$ of all death in Japan. Therefore, Brain dock is popular method to take preventive measures in Japan. In this Brain dock, there are two common diseases that are found. One is lacunar infarct and other is Enlarged Virchow-Robin space. Lacunar infarcts have the risk for developing stroke. On the other hand, Enlarged VirchowRobin space is low-risk disease. Distinguishing these two diseases is important task of Brain dock. However these two diseases have similar appearance on Magnetic Resonance (MR) images. Thus, doctors raise expectations for Computer Aided Diagnosis (CAD) of lacunar infarcts. We focused on favorite site of lacunar infarcts and made the probabilistic atlas for one of the feature of machine learning. The experimental results validate that the probabilistic atlas can improve the accuracy of lacunar infarcts.
\end{abstract}

Keywords-lacunar infarcts; probabilistic atlas; computer aided diagnosis; magnetic resonance image

\section{INTRODUCTION}

Ministry of Health, Labor and Welfare reports that more than 120,000 people died of the cerebral vascular disease in Japan. The cerebral vascular disease differs substantially from other disease in that it has risk of aftereffects. Therefore, The Brain dock is in widespread use in Japan to early detection and rapid cure. Two common diseases: lacunar infarcts and Enlarged Virchow-Robin space are found in this Brain dock. These diseases have similar appearance on Magnetic Resonance (MR) images. Consequently, there are two important problems.

These diseases are difficult to distinguish.

The diagnosis is subjective

Thus, doctors raise expectation for Computer Aided Diagnosis (CAD) for lacunar infarcts detection. Some researches about lacunar infarcts detection are reported [1][2].

We focused on favourite site of lacunar infarcts [3]. On the basis of anatomical feature, we propose that making and using the probabilistic atlas of lacunar infarcts for automatic detection. Additionally, we developed the software of lacunar infarcts detection to aspire for clinical application.

\section{AutOMATIC DEteCtion Of LACUNAR INFARCTS}

In our system, there are three steps for automatic detection of lacunar infarcts.

Step 1: Making the probabilistic atlas and amount of characteristics database of lacunar infarcts from study data sets

Step 2: Extracting initial candidate regions of lacunar infarcts by thresholding with intensity of MR image and volume

Step 3: Classifying lacunar infarcts and False Positives (FPs) using probabilistic atlas and Support Vector Machine (SVM)

\section{A. Making the Probabilistic Atlas of Lacunar Infarcts}

Step 1, we made the probabilistic according to the following steps.

1. Segmenting input data into three anatomies (gray matter, white matter, cerebrospinal fluid (CSF)) using VBM8[4]

2. Calculating deformation field to standard (MNI) brain from non-rigid registration using DARTEL[5]

3. Mapping gravity center of lacunar infarcts on MNI brain with the deformation field

\section{Convoluting with three dimensional Gaussian filter}

In this study, we used analysis tool that is called SPM8[6]. SPM8 has the many functions for brain image processing; we used VBM8 for segmentation and DARTEL for non-rigid registration.

Brain MR images have gap because of setting at the time of photography and differences among individuals of head configuration (Fig. 1 (a)). Figure 1 (b), shows overlay two MR images after non-rigid registration. As you can see two subjects have same configuration and this makes it possible to create high precision atlas.

Number of case $N$, patient Number $i=(1,2, \ldots, N)$, deformation field of patient Number $i \mathbf{F} i$, binary images of lacunar infarcts gravity center $\mathbf{L} i$. Then probabilistic atlas is derived from Eq. 1. 


$$
\operatorname{atlas}(x, y, z)=\sum_{i=1}^{N} F_{i}\left(L_{i}(x, y, z)\right)
$$

The filter size of three-dimensional Gaussian filter, we adopted $15 * 15 * 15$ voxels. As Fig. 2 shows the probabilistic atlas of lacunar infarcts that was generated in this study. Figure 2 (a) and (b) show the atlas at brain stem, thalamus and putamen in brain. The part of high intensity in the atlas, there are strong possibility of existing lacunar infarcts. The existing probability is one of the feature vectors for inputting to SVM clustering.

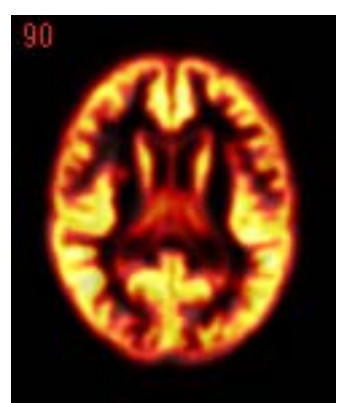

(a) Before non-rigid registration

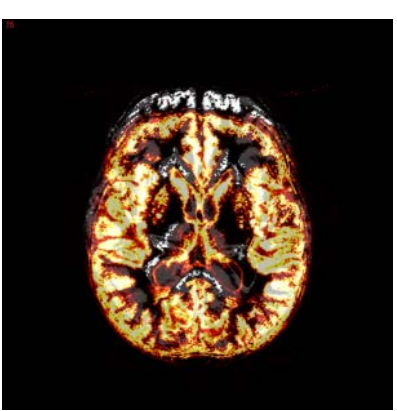

(b) After non-rigid registration
FIGURE I. OVERLAY MR IMAGES OF TWO SUBJECTS

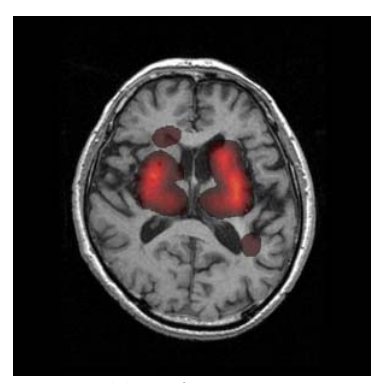

(a) Brainstem

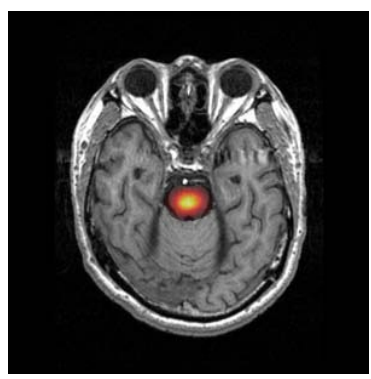

(b) Thalamus / Putamen
FIGURE II. LACUNAR INFARCTS PROBABILISTIC ATLAS.

\section{B. Extracting Initial Candidate Regions}

Step. 2, we extracted initial candidate regions of lacunar infarcts by thresholding using intensity of MR images and volume information. Intensity distributions of MR images are different because of the noise or setting of MR machine. Thus we normalized the intensity using Eq. 2.

$$
\mathbf{X}_{i_{-} \text {new }}=\mathbf{X}_{i_{-} \text {old }} / \mu_{i}
$$

In this equation, $\mathbf{x}_{i_{\text {oold }}}$ is original image of patient number $i$, $\mathbf{X}_{i_{-} \text {new }}$ is normalized image. After intensity normalization, we applied intensity binarization range of $0<\mathbf{X}_{i_{-} \text {new }}<0.85$. This range was derived from Fig.3. The figure shows distributions of lacunar infarcts and non-lacunar infarcts intensity and we decided a maximum range that point where the two distribution cross.

Next, the result binary image from before step was applied threshold by volume (number of coupled voxels) between 0 to 1,500 voxels. The result image is initial candidate region of lacunar detection. Threshold value for volume was empirically derived.

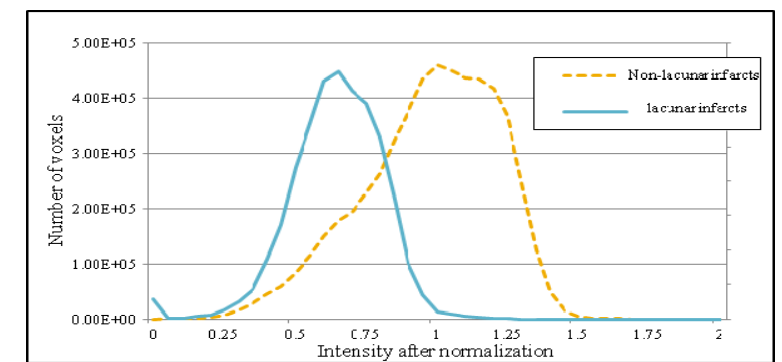

FIGURE III. DISTRIBUTION OF AVERAGE INTENSITY OF LACUNAR INFARCTS AND NON-LACUNAR INFARCTS REGIONS.

\section{Classification Lacunar Infarcts as False Positive Using} Svm with Atlas

Step. 3, we classified lacunar infarcts as FPs using SVM with the atlas. We inputted feature vector that contain three components to SVM.

Mean intensity of the initial candidate region

Volume (number of coupled voxels)

Lacunar infarcts probability from atlas

Lacunar infarcts probability was derived from the atlas value at gravity center of initial candidate region. We preliminarily calculated these features from study dataset and used for the database. SVM results were indicated by probability of lacunar infarcts.

\section{EXPERIMENT}

In this study, we used 106 MR images (3D T1-weighted images) that were provided from Shiga University of Medical Science. These images have one or more lacunar infarcts and voxels size are $0.47 * 0.47 * 1.00 \mathrm{~mm}$. These are made diagnosis by a doctor, and we used the diagnosis for gold standard in our study. In total, there are 299 lacunar infarcts.

Using probabilistic atlas that was made in Step 1, we derived initial candidate region by intensity and volume thresholding in Step 2. Result of this step, there were 257 lacunar infarcts and 14,884 FPs were found.

Secondly, we inputted the feature vector that contains intensity, volume and existence probability of lacunar infarcts to SVM.

Figure 4 shows classification accuracy of initial candidate region. The evaluation result was used Leave-one Out method. The Area Under the Curve (AUC) of proposed method and conventional method were 0.958 and 0.929 . Furthermore, we did the test of significant difference between two AUC [7]. As a result of this test, there is significant difference between result of proposed (with atlas) method and conventional (without atlas) method ( $p=0.0047<0.025)$. As the result, classification lacunar infarcts and FPs with atlas is strong result. 


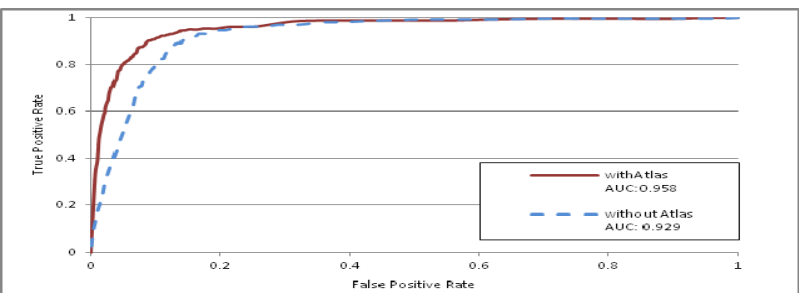

FIGURE IV. ROC CURVE OF CLASSIFICATION ACCURACY.

\section{Developing Software OF Computer Aided DIAGNOSIS}

We developed software of lacunar infarcts detection to aspire for clinical application. This software makes it possible to automatic detection of lacunar infarcts and used by many people who is not good at PC. It use the probabilistic atlas and database of features for automatic detection.

Figure 5 shows display of result that was detected by this software. There are two display modes. One shows input image and result image (Fig. 5 (a)). The other shows input image with atlas and result image (Fig.5 (b)). In the result image, it shows probability by colors. If there is high probability of lacunar infarcts, then there regions would be red, and low probability, it shows blue. These modes are able to change by the tabs.

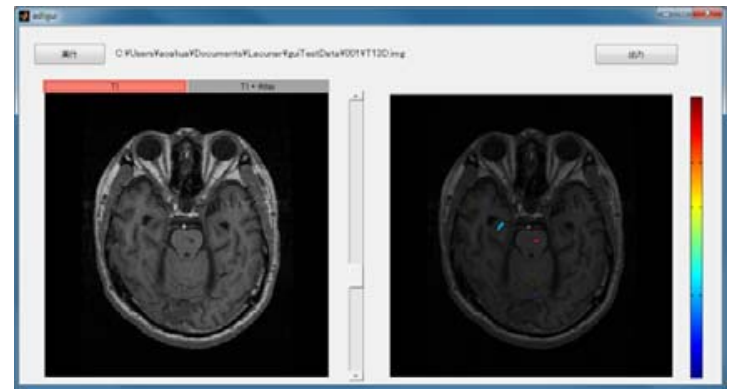

(a) Input and result image.

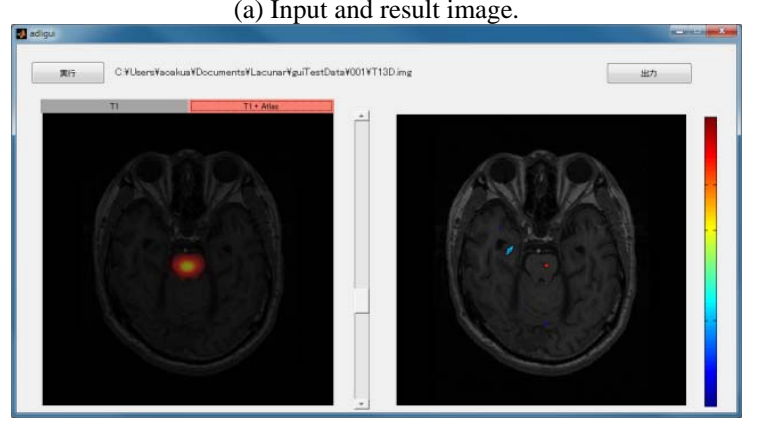

(b) Atlas and result image.

FIGURE V. DISPLAY RESULT OF SOFTWARE.

\section{CONCLUSION}

In this paper, we proposed the lacunar infarcts atlas for classifying lacunar infarcts and FPs. As a result, we could improve the accuracy of lacunar infarcts detection using the atlas as one of feature for SVM. Furthermore, we developed software of lacunar infarcts detection to aspire for clinical application. In the future, we try to improve accuracy of extraction of initial candidate region, also use images that was taken other imaging method of MR to add features.

\section{ACKNOWLEDGMENT}

This work is supported in part by the Grant-in Aid for Scientific Research from the Japanese Ministry for Education, Science, Culture and Sports (MEXT) under the Grant No. 2430076 in part by the MEXT Support Program for the Strategic Research Foundation at Private Universities (20132017), and in part by the R-GIRO Research Fund from Ritsumeikan University.

\section{REFERENCES}

[1] Tanigawa F, Uchiyama R, Matsumura C, et al., "Improvement of CAD scheme for detection of lacunar infarcts in MR images using AdaBoost template matching,” IEICE Technical Report, Vol. 117, pp. 323-326, 2014. In Japanese.

[2] Gatayama K, Kashima M, Sata K, et al., "Research on Automatic Detection of Lacunar Infraction in Brain CT Images: Configuration of Detection Areas by Level Set Method,” IEICE Technical Report, Vol. 109(470), pp. 317-322, 2010. In Japanese

[3] Longstreth WT Jr, Bernick C, Manolio TA, et al., "Lacunar infarcts defined by magnetic resonance imaging of 3660 elderly people: the Cardiovascular Health Study,” Arch Neurol, Vol. 55, pp. 1217-25, 1998.

[4] Ashburner J, Friston KJ, “Unified segmentation,” Neuroimage, Vol. 26(3), pp. 839-851, 2005.

[5] Ashburner J, “A fast diffeomorphic image registration algorithm," NeuroImage, Vol. 38, pp. 95-113, 2007.

[6] “Statistical Parametric Mapping (SPM),” UCL Institute of Neurology, http://www.fil.ion.ucl.ac.uk/spm/, Last Modify, 2014/07/29.

[7] Hanley JA, McNeil BJ, "A method of comparing the areas under receiver operating characteristic curves derived from the same cases," Radiology, Vol. 148(3), pp. 839-843, 1983. 